\title{
La situación de la investigación y la formación en turismo y estudios lingüísticos en España
}

\author{
Sergio Rodríguez-Tapia \& Adela González Fernández \\ Universidad de Córdoba \\ sergio.rodriguez@uco.es \& adela.gonzalez@uco.es \\ https://dx.doi.org.10.12795/futhark.2019.il4.13
}

Fecha de recepción: 10.11 .2018

Fecha de aceptación: 20.01 .2019

Resumen: En este estudio se pretende conocer la situación actual de la investigación y la formación de los estudios universitarios que integran turismo y lenguas en España, partiendo de la relevancia con la que cuenta el sector turístico en este país y teniendo en cuenta la importancia de la formación e investigación en estudios lingǘsticos de aquellos profesionales que se dedican a este sector. Para estudiar la investigación, se han recopilado 367 trabajos de investigación entre 1967 y 2018 a partir de Dialnet con la conjunción de diversas palabras clave. Para analizar la formación (principalmente los estudios de Turismo y de Traducción e Interpretación), se ha empleado la base de datos del RUCT para identificar los tipos de asignaturas que se incluyen en todos los planes de estudio. Como conclusión, se esboza que la preocupación investigadora en torno al turismo desde una perspectiva lingüística ha aumentado desde 2016.

Palabras clave: estudios; formación; traducción; turismo; universidad; investigación.

\section{The Situation of Research and Education in Tourism and Linguistic Studies in Spain}

\begin{abstract}
The aim of this study is to ascertain the current situation of research and university studies integrating tourism and languages in Spain, based on the relevance of the tourism sector in this country and taking into account the importance of training and research in linguistic studies for professionals working in this sector. In order to study the research aspects, 367 research papers have been compiled between 1967 and 2018 from Dialnet, with the conjunction of several key words. To analyse the situation of the education studies (mainly Tourism and Translation and Interpretation studies), the RUCT database has been used to
\end{abstract}


identify the types of subjects included in all curricula. In conclusion, it is outlined that research concern about tourism from a linguistic perspective has increased since 2016 .

Keywords: education; studies; translation; tourism; university; research.

Sumario: I. Introducción: marco económico del turismo en España. 2. Necesidad de los estudios lingüisticos y la traducción turistica en España. 3. Metodología. 4. Investigación en turismo y estudios lingüisticos en España. 4.I. Años de publicación. 4.2. Tipos de publicaciones. 4.3. Enfoques de los estudios. 4.4. Objetos temáticos de los estudios. 4.5. Lenguas de los estudios. 5. Formación en turismo y estudios lingüísticos en España. 5.I. Estudios sobre Traducción e Interpretación. 5.2. Estudios sobre turismo. Conclusiones.

\section{Introducción: marco económico del turismo en España}

El sector turístico en España es uno de los principales pilares del desarrollo económico nacional, no solo por el impacto directo que supone, sino también por el efecto indirecto que ejerce sobre otros sectores de la economía. Además, este sector juega un papel fundamental en la creación de empleo y en los ingresos por exportaciones, y contribuye de forma directa, según datos de la OCDE (OECD 2018), a una media del $4,2 \%$ del Producto Interior Bruto, del $6,9 \%$ del empleo y del $21,7 \%$ de las exportaciones en los países de la OCDE. Estas cifras promedio pertenecientes a este conjunto de países aumentan considerablemente en el caso particular de España, donde encontramos que la actividad turística, además de ser uno de los sectores económicos más importantes e impulsor del desarrollo, representa el II,I \% del Producto Interior Bruto del país y el $13 \%$ del empleo, contribuyendo así a paliar el déficit de mercado con unas cifras que alcanzan 54,5 mil millones de euros, un $7,1 \%$ más que en 2015.

En el mismo informe de la OCDE, se informa de que España es el tercer destino turístico a nivel mundial y el segundo en términos de ingresos, con 75,3 millones de llegadas de turistas. Estos datos, unidos al hecho de que los mercados chino y ruso están aumentando anualmente debido a las mejoras en las infraestructuras, en las comunicaciones y en las relaciones diplomáticas, permitieron que el Foro Económico Mundial declarara a España en 2017, por segundo año consecutivo, el primer país en términos de competitividad para el turismo (OECD 2018).

Según el Instituto de Turismo de España, organismo perteneciente al Ministerio de Energía, Turismo y Agenda Digital del Gobierno de España (TURESPAÑA 2018), las estimaciones en cuanto al crecimiento de la entrada en España de turistas internacionales y del gasto total asociado son positivas en cuanto al crecimiento. Se prevé que las llegadas alcanzarán el 2,2 \% y el gasto total el 4,4 \%, 
lo que equivale a más de 23 millones de turistas y 23,5 mil millones de gasto. A estas previsiones hay que sumar, además, un mantenimiento del crecimiento en el número de afiliados a la Seguridad Social vinculados al turismo.

En lo que a la demanda se refiere, esta ha seguido creciendo en el primer semestre de 2018 por parte de los no residentes, manteniendo así una tendencia positiva que se suma a las notables subidas de los dos años anteriores. Por otro lado, desde la perspectiva del empleo, el informe del primer trimestre de TURESPAÑA (2018) asegura que las actividades turísticas siguen siendo uno de los principales creadores de empleo, a la vez que están contribuyendo de forma decisiva al fortalecimiento de la economía española. De este modo, este año 2018 ha comenzado con cifras positivas y crecimientos en turismo, viajes y balanza de pagos, aunque esta última está registrando una senda de ligero decrecimiento con respecto a los últimos meses del año pasado. Aun así, los pagos por turismo continúan siendo inferiores a los ingresos.

Las previsiones para el segundo semestre de 2018 son de moderado crecimiento y se espera que se superen las cifras del mismo semestre del año anterior, gracias, entre otros aspectos, a la confianza en que las llegadas de turistas internacionales crezcan en torno al 2,2 \%, superando así la cifra de 23 millones de visitantes de fuera de nuestras fronteras (TURESPAÑA 20I8). En este panorama de crecimiento en términos globales, el sector turístico se ve reforzado por inversiones a nivel local, regional y nacional que afectan a distintos ámbitos de la sociedad y del desarrollo, desde cuestiones medioambientales hasta aspectos relacionados con la sociedad del conocimiento. Por ello, consideramos que las universidades, como principales generadoras de desarrollo y transmisoras de conocimiento, no deben quedarse atrás en esta tendencia social, política y económica crecientes en la que todos los agentes se encuentran inmersos.

En esta misma línea, la Lingüística y las disciplinas académicas afines comienzan, aunque con cierta timidez, a abrirse camino en nuevos contextos docentes e investigadores. En este trabajo nos proponemos como objetivo llevar a cabo un análisis descriptivo del panorama en la investigación y en la formación, en el marco de las universidades españolas, de los estudios relacionados con el turismo y las lenguas, para así poder determinar el grado de actualización de estos y su adecuación con respecto a las demandas de una sociedad cambiante y exigente en este contexto.

\section{Necesidad de los estudios lingüísticos y la traducción turística en España}


A la luz de los datos expuestos en la sección previa, parece obvio que el contacto entre distintas culturas y entre distintas lenguas es inherente a la situación social y económica en la que nos encontramos inmersos. En este contexto, una correcta labor lingüística en general y traductológica en particular que posibilite la comunicación entre distintos agentes en cualquier situación resulta fundamental.

Al igual que ocurre en otros ámbitos, como por ejemplo el agroalimentario (Moreno y Rodríguez-Tapia 2015), se hace patente la necesidad de una formación lingüística global especializada en el campo del turismo para el buen funcionamiento de la sociedad y de los mercados. También Porlán (2013) refleja esta situación en su entrevista a la Area Manager de COVAP, Ana Ballesteros, cuando esta afirma que no solo el conocimiento del inglés resulta clave en el desarrollo de la economía, sino también de otras lenguas a priori más lejanas para nosotros, como pueden ser el chino o el ruso.

Según datos de TURISCOR (2018), a finales de 2017 y a principios de 2018 , los principales mercados emisores de turistas para España fueron Alemania, Italia, los países nórdicos, Reino Unido y Francia. Cada uno de ellos con distintas cifras y con distintas tasas interanuales $y$, a pesar de que algunos, como los dos últimos, muestran tasas de desaceleración, los gastos medios mantienen una senda positiva. Por otro lado, como ya hemos mencionado anteriormente, se prevé una mejora en el segundo semestre de 2018 con respecto al mismo período de 2017 y se espera que aumente el turismo proveniente de Irlanda y los países nórdicos, de los países del sur de Europa, del norte de América y de lberoamérica. Además, se prevé un mantenimiento destacable en el número de turistas provenientes de Centroeuropa y de Europa del Este y, especialmente, de los mercados de Asia-Pacífico, en concreto del chino.

En este contexto de internacionalización del turismo y del contacto entre un repertorio tan numeroso de lenguas, consideramos que para los estudios lingüísticos $y$, en concreto, para los estudios de traducción, es fundamental una especialización en el ámbito turístico, en la que se preste especial atención al léxico, al discurso y a las necesidades comunicativas específicas del sector. Asimismo, y de manera inversa, los estudios relacionados con el turismo no pueden considerarse completos sin una formación específica en lenguas que posibilite a los futuros profesionales del sector una comunicación correcta y eficaz.

Por ello, en este trabajo llevamos a cabo un análisis de la situación de la investigación y de la formación en turismo y en lenguas en nuestro país con el objetivo de realizar un diagnóstico de la situación y plantear la posibilidad de prestar una mayor atención a este tipo de estudios, en caso de que fuera necesaria. 


\section{Metodología}

Para realizar de este trabajo se ha seguido una metodología dividida en varias fases $y$ en dos direcciones de forma paralela. Es decir, puesto que nuestro objetivo es conocer la situación, desde una perspectiva descriptiva, no solo de los estudios lingüísticos (fundamentalmente de Traducción e Interpretación, puesto que son los que suelen contar con contenidos relacionados con el turismo) y de Turismo en España, sino también la de la investigación en estos ámbitos, se ha llevado a cabo un proceso doble: se han seguido los mismos pasos tanto para la parte de la formación como para la de investigación, para así extraer resultados individuales y poder contrastarlos posteriormente. Conviene aclarar que los estudios de investigación analizados para este trabajo han sido seleccionados teniendo en cuenta que hubieran sido publicados en libros o revistas españoles o, en el caso de que se tratara de ediciones extranjeras, que los investigadores fueran españoles.

La primera fase de nuestro estudio ha consistido en realizar varias consultas en bases de datos y repositorios bibliográficos. Para conocer el número, por un lado, de estudios oficiales relacionados con turismo y con traducción se ha consultado la base de datos RUCT' (Registro de Universidades, Centros y Títulos), creada por el Ministerio de Educación, Cultura y Deporte del Gobierno de España. A la hora de realizar la búsqueda, se han filtrado todos aquellos estudios de Traducción e Interpretación y todos aquellos estudios de Turismo que se encontraran íntimamente relacionados con estos. Con el objetivo de obtener resultados lo más precisos posible, se han tenido en cuenta los estudios pertenecientes a los niveles de grado, máster y doctorado. Tras identificar y seleccionar todas las titulaciones de Turismo, se ha llevado a cabo un análisis minucioso de cada plan de estudios y se han filtrado las materias siguiendo los siguientes parámetros:

a) Asignaturas que no incluyen estudios lingüísticos.

b) Asignaturas que incluyen estudios de lengua instrumental.

c) Asignaturas que incluyen estudios relacionados con las habilidades comunicativas.

d) Asignaturas que incluyen otros estudios relacionados con las lenguas y la comunicación.

' Puede consultarse en el siguiente enlace: https://www.educacion.gob.es/ruct/home. 
El mismo procedimiento se ha seguido con las titulaciones de Traducción e Interpretación, donde se han buscado las materias que tuvieran alguna relación el turismo y se han clasificado según contasen con:

a) Asignaturas que no incluyen la traducción turística en su planificación.

b) Asignaturas que versan total $\circ$ parcialmente sobre traducción turística.

c) Asignaturas que incluyen la traducción turística o algún contenido sobre turismo de forma explícita en sus guías docentes.

Una vez finalizado el vaciado de titulaciones y clasificadas según estos parámetros, se procedió a efectuar la búsqueda bibliográfica para determinar en qué punto se encuentra la investigación académica relacionada con esta temática. En este punto, se realizaron búsquedas combinadas en dos bases de datos bibliográficas: Dialnet ${ }^{2}$ — la base de datos de la Universidad de la Rioja- y BITRA ${ }^{3}$ —perteneciente al Departamento de Traducción e Interpretación de la Universidad de Alicante. Las búsquedas se realizaron en marzo de 2018 con una serie de palabras clave extraídas de una investigación previa (González-Fernández y Rodríguez-Tapia 2018), en la que un grupo constituido por trece informantes, todos ellos alumnos de Traducción e Interpretación de la Universidad de Córdoba, seleccionaron todas las unidades léxicas especializadas de diversos textos pertenecientes a ocho tipologías textuales distintas (informe técnico, guía turística, folleto, artículo científico, artículo periodístico, sitio web, contrato de servicio y reglamento o normativa), todos ellos relacionados con el campo del turismo. El corpus obtenido con todas estas unidades léxicas ha sido analizado para este estudio con la herramienta de gestión de corpus textuales AntConc, gracias a la cual se ha extraído una lista de frecuencias para obtener las unidades con un mayor índice de aparición en la totalidad de los textos. Una vez realizada esta operación, obtuvimos las siguientes unidades léxicas como las más frecuentes: turismo, tour, contrato, viaje, viajero, turista, negocio, hotel, precio, servicio, alojamiento, internacional, transporte, destino, actividades, cancelación, visitante, operador, evento y turístico. Para poder encontrar investigaciones que trataran conjuntamente aspectos del ámbito turístico con aspectos lingüísticos, cruzamos estos términos con los siguientes: traducción, lengua, diccionario, vocabulario, léxico, glosario, lexicografia, terminología y terminografia. Las búsquedas se llevaron a cabo combinando todos los términos entre sí y en los idiomas español e inglés.

\footnotetext{
2 Puede consultarse en el siguiente enlace: https://dialnet.unirioja.es/.

${ }^{3}$ Puede consultarse en el siguiente enlace: http://aplicacionesua.cpd.ua.es/tra_int/usu/buscar.asp.
} 
A continuación, tras el vaciado de textos, estos fueron clasificados según el año al que pertenece la publicación, el tipo o formato de publicación, el enfoque de trabajo, el objeto de estudio y las lenguas en las que se centra.

En los siguientes apartados procederemos a exponer los resultados obtenidos de las búsquedas y el análisis de la información obtenida.

\section{Investigación en turismo y estudios lingüísticos en España}

\section{I. Años de publicación}

Se recopilaron un total de 367 trabajos de investigación, que abarcan desde el año 1967 al 2018. Como muestra la Figura I, 2016 resulta el año más productivo en la investigación lingüística en torno al turismo, destacando muy por encima de otros años productivos, como 2012 o 2014. En 2016 se publicaron 26 artículos en revistas científicas y 7 capítulos de libro, además de tratarse del año en el que más tesis doctorales sobre dicho tema fueron publicadas: un total de 9 tesis doctorales, siendo la enseñanza de lenguas en el entorno turístico, especialmente la del español, el tema principal de análisis. No obstante, no parece que exista una relación estrecha entre los objetos de estudio o los temas de la tesis, lo que hace pensar que no pertenecen a una línea de investigación conjunta ni son fruto de un proyecto común.

Desde 2005, la producción científica en este campo ha superado la media de publicaciones en los 31 años que existen con publicaciones (es decir, II publicaciones por año), casi siempre duplicando dicho promedio, lo que hace considerar la relevancia académica que ha ganado en los últimos lustros la investigación en torno al turismo y sus discursos. 


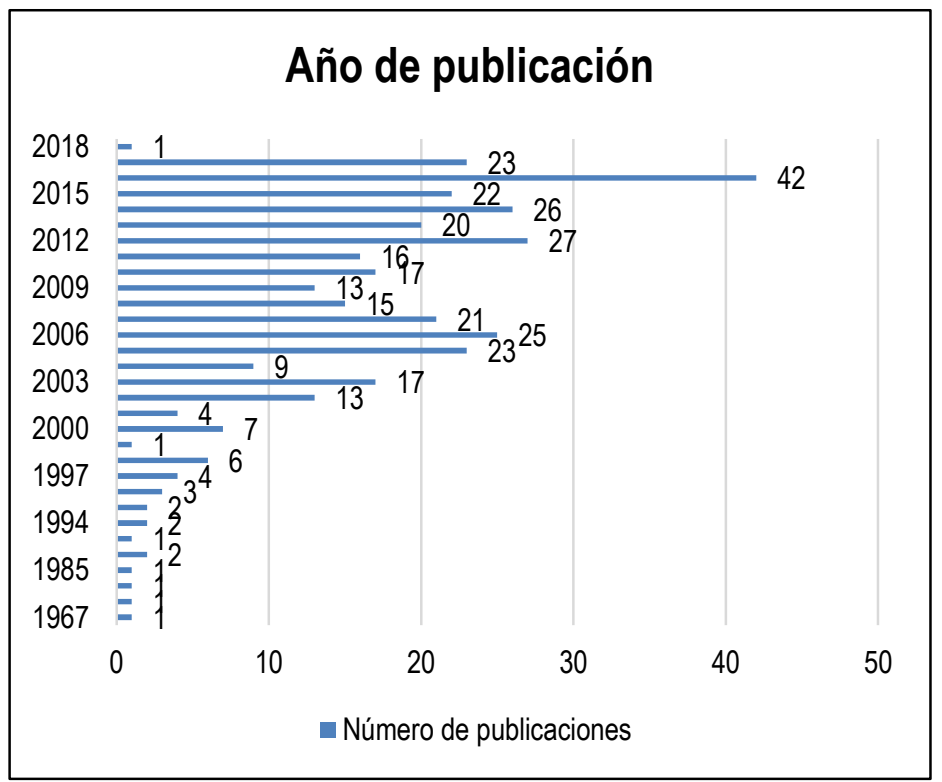

FIGURA I: Relación de años de publicación. Fuente: elaboración propia

\subsection{Tipos de publicaciones}

En cuanto al formato en el que se publican los trabajos (véase Figura 2), destacan los artículos en revistas científicas y los capítulos de libro, siendo los primeros los que alcanzan casi la mitad del total de publicaciones (176 de 366 publicaciones).

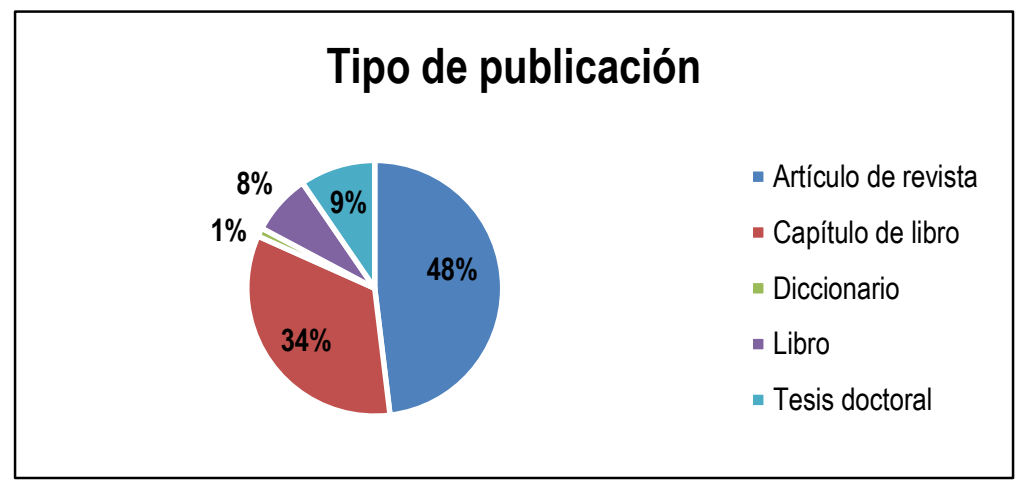

FIGURA 2: Relación de tipo de publicaciones. Fuente: elaboración propia 
Buena parte de estas publicaciones (62 trabajos) tienen carácter lingüístico (relacionado con los discursos, la contrastividad, el léxico, etc.), seguidos de 49 trabajos de corte traductológico, 37 de enseñanza de lenguas, 18 con carácter terminológico y 10 relacionados con la didáctica de algún aspecto relacionado con la cultura o la traducción.

Si tenemos en cuenta los libros publicados ( $8 \%$ ), destaca el hecho de que 23 de los 28 libros publicados son previos a 2010. Además, destacan los trabajos de enseñanza de lenguas, especialmente del alemán y del inglés. A partir de 2010, y hasta 2014, solo se han publicado 5 libros, de los cuales destacan el enfoque traductológico, aunque también existen contribuciones sobre terminología o enseñanza de lenguas.

Con respecto al porcentaje más escaso (I \%), que corresponde con los diccionarios, coinciden con los analizados en González-Fernández y Rodríguez Tapia (2018), es decir, con Montaner (1998), Deltoro (2000) y Alcaraz (2006), a los que se suma el diccionario turístico trilingüe de Salas (1989), que sirven como descripción de la escasez de recursos lexicográficos y terminográficos sobre turismo para el español.

\subsection{Enfoques de los estudios}

Para abordar los enfoques de estudio de las investigaciones recopiladas, hemos decidido emplear cinco grandes categorías: a) didáctico, si el trabajo se centra en enseñar cómo se traducen ciertos elementos o sobre métodos de enseñanza del léxico, la gramática o la cultura, es decir, si se centra en las competencias docentes; b) enseñanza de lenguas, si la publicación versa sobre la enseñanza-aprendizaje de componentes lingüísticos; c) lingüístico, se constituye como una categoría amplia en la que caben las reflexiones en torno a la gramática, las relaciones entre paradigmas lingüísticos, la contrastividad entre lenguas, etc.; d) terminológico, si se tratan los aspectos relacionados con la comunicación especializada; y e) traductológico, si el enfoque se realiza desde el trasvase de información entre lenguas y las correspondencias semántico-formales entre ellas (véase Figura 3). 


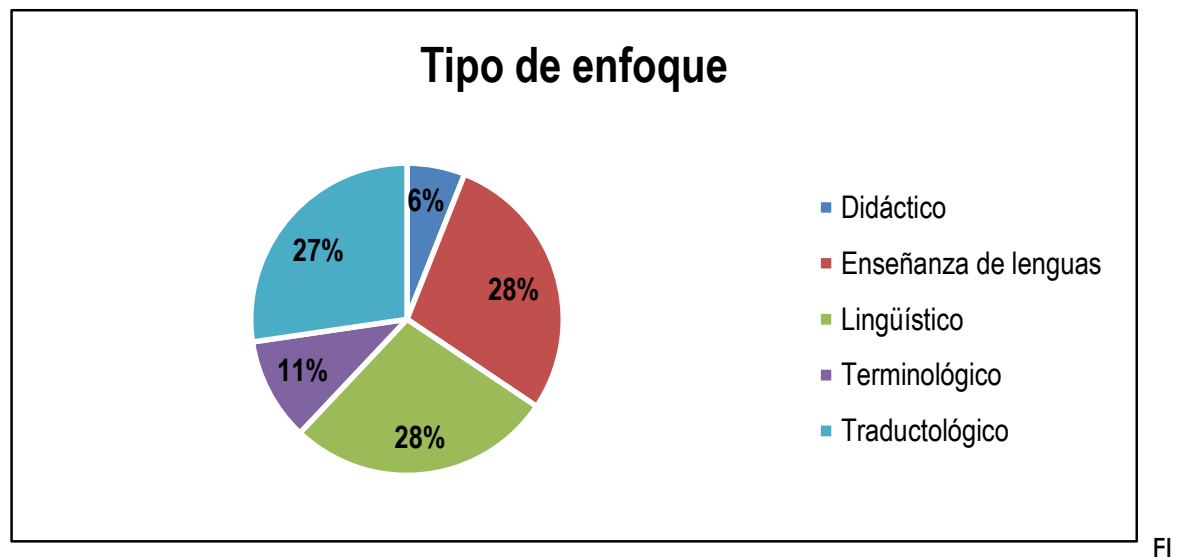

GURA 3: Relación de tipos de enfoques de estudio. Fuente: elaboración propia

El enfoque más destacado es el de enseñanza de lenguas, con un $28 \%$ sobre el total. Las lenguas más abordadas son el español, el alemán y el inglés. El año con más publicaciones sobre enseñanza de lenguas es 2016 ( 14 publicaciones), seguido de 2006, 2003 y 2002, con 9 publicaciones cada uno. Con respecto a los objetos de enseñanza, la gramática y los elementos relacionados con el significado son los más discutidos (57 trabajos), seguidos de la aplicación de las TIC a la enseñanza de lenguas ( 13 trabajos) o los elementos léxicos y discursivos ( 9 trabajos cada uno).

El enfoque lingüístico, que es el más general y abarcador, sigue al enfoque de enseñanza de lenguas. El año más productivo también coincide con 2016 (con un total de 17 trabajos) y los objetos más abordados son la lengua, en especial la gramática (37 trabajos), seguida del discurso (22 publicaciones) y el léxico (I I publicaciones).

Por último, los trabajos con enfoque traductológico ( $27 \%$ sobre el total) centran sus estudios en las lenguas española, inglesa y alemana. Los años más productivos son 2014 y 2012 , en los que se analiza, sobre todo, el léxico, la cultura, la literatura de viajes y la traducción ( $9,10,1$ I y 34 trabajos respectivamente).

\subsection{Objetos temáticos de los estudios}

En el caso de los objetos temáticos (véase Figura 4), destacan las publicaciones que versan sobre la lengua, entendida esta como código, en muy estrecha relación con la enseñanza de lenguas, o como interfaz entre paradigmas lingüísticos. El léxico y la traducción son los otros dos objetos más analizados en nuestro corpus. Por una parte, el léxico se plantea como clave en el discurso 
turístico, desde su caracterización como discurso marcado por una temática que es interdisciplinar, y tiende a establecer relaciones con el ámbito administrativo, jurídico, económico, social, lúdico o gastronómico. Por otra parte, los estudios dedicados a la traducción se centran en la formulación en lengua meta de estructuras fraseológicas específicas, el léxico o discursos novedosos.

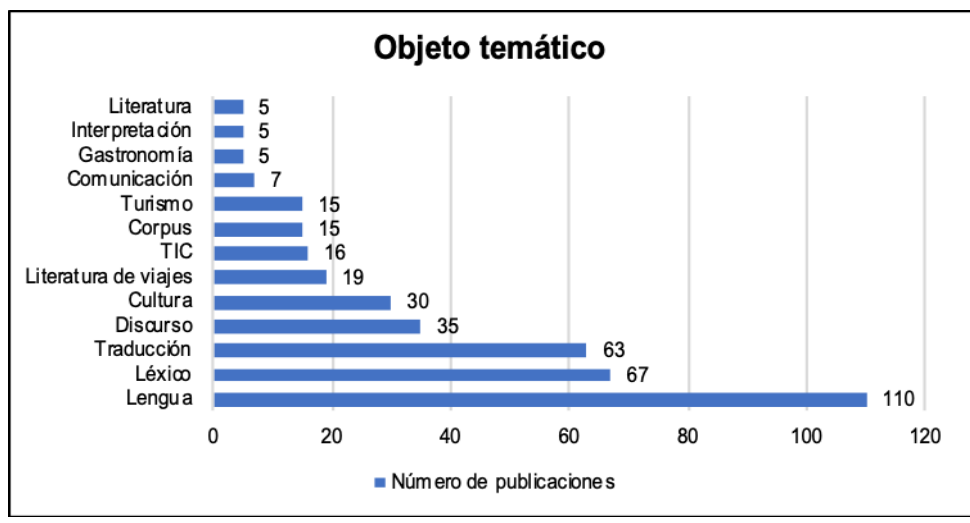

FIGURA 4: Relación de objetos temáticos. Fuente: elaboración propia

Dada la dificultad de establecer un único objeto temático en los estudios, muchos han sido clasificados de acuerdo con varios objetos, que han sido contabilizados sobre el total, de forma que una misma publicación puede contar con diversos objetos de estudio simultáneos, como, por ejemplo, el léxico, el discurso y la cultura.

\subsection{Lenguas de los estudios}

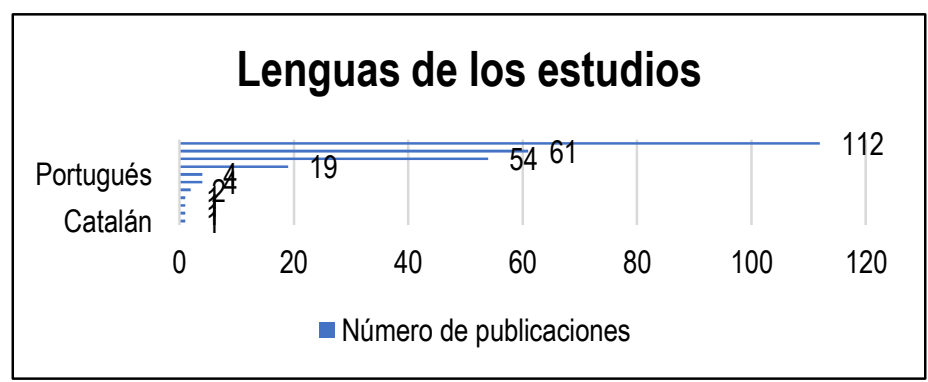

FIGURA 5: Relación de lenguas. Fuente: elaboración propia 
Las lenguas más abordadas coinciden con lo expuesto en apartados anteriores, en los que el español, el inglés y el alemán son las lenguas a las que más investigación se ha dedicado en el marco de los estudios lingüísticos en torno al turismo (véase Figura 5). Es posible que la atención prestada a estas lenguas sea fruto de la necesidad de cubrir ciertas carencias formativas (principalmente se trata de estudios desde la enseñanza de lenguas y desde la traducción) o bien de profundizar en la caracterización del código discursivo del turismo. Como se puede observar, el resto de lenguas, entre las que se incluyen el francés, el portugués, el italiano o el chino, no ha recibido especial atención en los estudios científicos en el panorama nacional.

\section{Formación en turismo y estudios lingüísticos en España}

Para facilitar el análisis descriptivo del conjunto de titulaciones que han sido investigadas, se ha dividido esta sección en dos partes, cada cual conformada por un gráfico con los resultados del estudio y una tabla con los tipos de asignaturas, que sirve de leyenda.

\section{I. Estudios sobre Traducción e Interpretación}
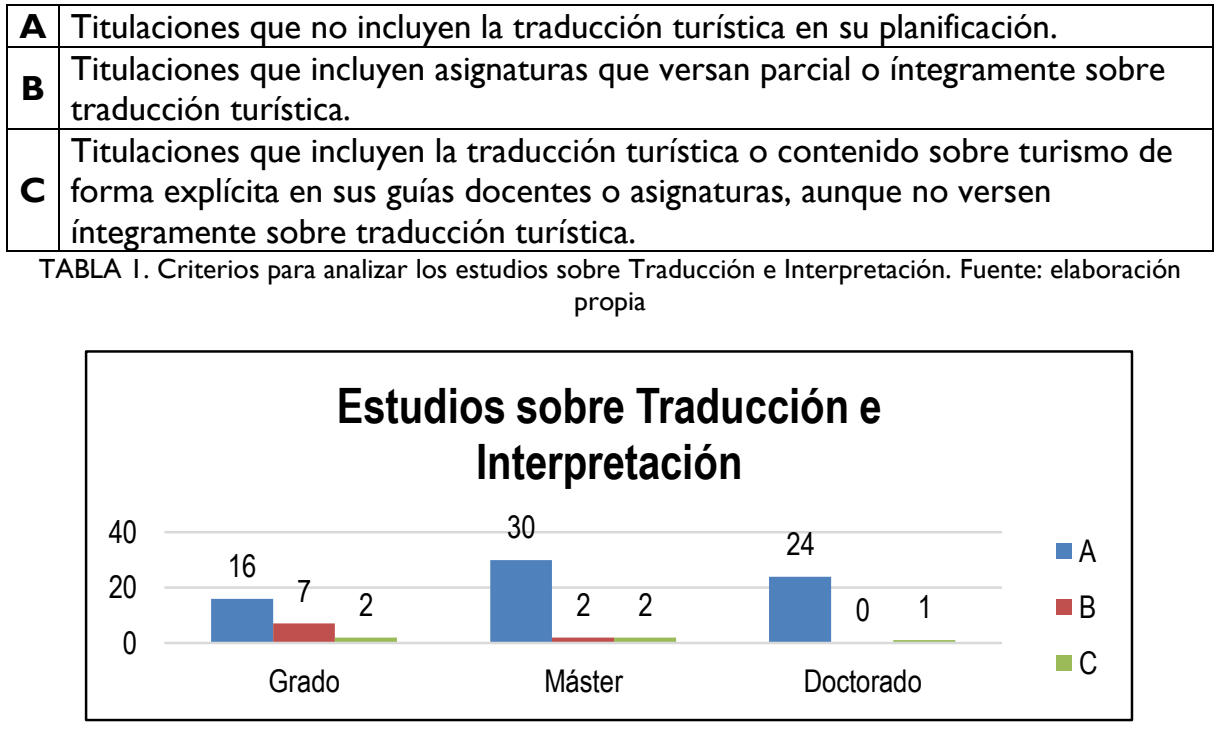

FIGURA 6: Relación de estudios sobre Traducción e Interpretación. Fuente: elaboración propia 
La gran mayoría de las titulaciones de Traducción e Interpretación no cuentan con formación en traducción turística o relacionada. De los 25 grados estudiados, solo 9 (el $36 \%$ ) incluyen el turismo en su planificación; en el caso de máster 30 de 34 titulaciones no cuenta con formación en traducción turística y solo I de los 25 programas de doctorado plantea dicho campo (véase Figura 6).

En el caso de las titulaciones de grado que incluyen asignaturas que versan parcial o íntegramente sobre traducción turística se incluyen las universidades de Alfonso X El Sabio, Córdoba, Las Palmas de Gran Canaria, Murcia, Salamanca y Valladolid. Otras, como la Universidad San Jorge o la Universidad de Granada, indican en algunas guías docentes que "se trabajará con el turismo y los museos" o se recomienda bibliografía relacionada sobre traducción y texto turísticos.

Si abordamos los estudios de máster, solo dos universidades (la Universidad de Córdoba y la Universidad de Vigo) incluyen asignaturas como "La traducción de textos pertenecientes al sector de las comunicaciones", "Traducción de textos turísticos" o "Traducción y patrimonio", en el cual existe un bloque de contenidos que se denomina "traducción y turismo".

Solo el programa de doctorado de la Universidad de Málaga deja intuir la línea de trabajo en traducción turística dentro de la línea de investigación "Traducción especializada, tecnologías de la traducción y la interpretación".

\subsection{Estudios sobre turismo}

D Titulaciones que no incluyen estudios lingüísticos en su planificación.

E Titulaciones que incluyen estudios de lengua instrumental en su planificación.

F Titulaciones que incluyen estudios relacionados con habilidades comunicativas en su planificación.

G Titulaciones que incluyen otros estudios relacionados con las lenguas y la comunicación.

TABLA 2. Criterios para analizar los estudios sobre Turismo. Fuente: elaboración propia 


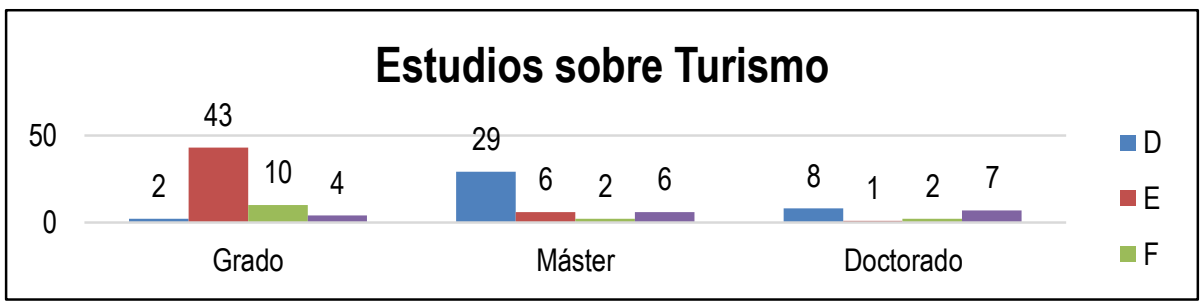

FIGURA 7: Relación de estudios sobre Turismo. Fuente: elaboración propia

En el caso de los estudios sobre Turismo, las asignaturas de algunas titulaciones han sido clasificadas en varias categorías, de forma que computan en todas ellas. En este trabajo se ha analizado un total de $1 / 2$ titulaciones relacionadas con el Turismo: 48 de Grado, 43 de Máster y 21 de Doctorado (véase Figura 7).

En el caso de Grado, el 90 \% de los estudios cuentan con asignaturas sobre lengua instrumental en su planificación, entre las que destacan el inglés, el francés, el alemán y el chino. De hecho, la amplia mayoría de los grados en Turismo cuenta con formación lingüística. Además, el $13 \%$ de los grados cuenta con formación en habilidades comunicativas y otras relacionadas, como se observa en las siguientes titulaciones:

- Universitat Politècnica de València: "Estrategias de comunicación para destinos turísticos" e "Intercultural Communication".

- Universidad de Murcia: "Técnicas de Comunicación” y "Traducción y Redacción de Textos Turísticos en Lenguas Extranjeras”. Destaca el hecho de que la Universidad de Murcia sea la única universidad española que oriente una de sus asignaturas a la redacción y traducción inversas.

En el caso de otras asignaturas relacionadas con la lengua y la comunicación, encontramos los ejemplos siguientes:

- Universidad de Oviedo: "Inglés I: Habilidades Sociales en el Entorno Turístico” e "Inglés II: Documentación Profesional para el Turismo”.

- Universidad de Cádiz: "Habilidades de comunicación interpersonal en el entorno turístico" y "Relaciones interculturales en el entorno profesional”.

- Universidad de Murcia: "Informador Turístico" y "Relaciones Públicas”.

El caso de máster y doctorado contrasta con la formación en grado. El $67 \%$ de los estudios de máster no cuenta con formación lingüística, siendo, tan solo, el $5 \%$ los que orientan sus estudios a la formación en lengua instrumental (Universidad Politécnica de Cartagena, de La Laguna, de Zaragoza y de Sevilla) y el $7 \%$ a otras habilidades comunicativas (Universitat de València, Católica de Valencia 
San Vicente Mártir, de Girona, de Málaga y Politécnica de Madrid) con asignaturas como "Gestión y marketing intercultural", "Writing skills for tourism", "Oral skills for tourism", "Comunicación, promoción y comercialización del turismo cultural", "Del marketing de destinos al marketing de producto" o "Imagen y Branding de los Destinos".

En el caso de doctorado, 10 de las 21 titulaciones contienen formación en habilidades comunicativas, muy estrechamente relacionadas con la internacionalización y el marketing.

\section{Conclusiones}

Teniendo en consideración los resultados analizados sobre la investigación, debe subrayarse que esta ha aumentado en gran medida en los últimos años, siendo 2016 el año con mayor número de trabajos. Este interés investigador también se observa en el mayor número de tesis doctorales desarrolladas en el último lustro, que revelan una mayor preocupación académica por el sector turístico y su conexión con la lingüística. No obstante, ha decrecido el número de monográficos sobre estudios lingüísticos en torno al turismo.

En la investigación, el objeto más abordado es, además de la propia gramática del discurso turístico, el léxico, que se estudia tanto desde el punto de vista traductológico como desde el punto de vista de la descripción microestructural. El hecho de que el léxico sea uno de los intereses académicos más frecuentes revela la caracterización temática a través del vocabulario del discurso turístico. No obstante, consideramos que debería profundizarse en otros asuntos discursivos o en la caracterización textual de todos los discursos sobre el turismo y extender las publicaciones de enseñanza de lenguas a otras lenguas diferentes que carecen de atención académica suficiente y que resultan relevantes en el contexto del sector turístico español.

En cuanto a la formación, contrasta la dedicación de los estudios de Turismo a la formación lingüística con la de los estudios de Traducción e Interpretación a los discursos turísticos. Por una parte, puede afirmarse que entre la organización de los planes de estudio sobre Turismo existe conciencia de la importancia de las habilidades lingüisticas y competencias comunicativas para los profesionales del sector turístico, lo cual se observa en el elevado porcentaje de titulaciones de grado que cuentan con asignaturas para la capacitación lingüística en diferentes lenguas; además, de carácter muy variado. A pesar de que en los estudios de máster y doctorado la dedicación formativa en lenguas se reduce, siguen existiendo titulaciones con formación en lenguas y comunicación. 
La formación en Traducción e Interpretación en turismo contrasta con la dedicación formativa mencionada para los estudios de Turismo. Tanto en los estudios de grado como en los de máster y doctorado son más numerosas las titulaciones que no cuentan con formación en traducción turística, siendo escasas las que se dedican íntegra o parcialmente a este campo. Este hecho también contrasta con la relevancia del turismo en España y con las necesidades comunicativas del sector turístico. A nuestro juicio, la única explicación plausible para esta ausencia de contenidos formativos en traducción turística se debe al amplio campo de la traducción, que dedica parte de sus estudios a intentar cubrir el máximo número posible de campos temáticos e instrumentales en los que la traducción tiene relevancia profesional. Aun así, debe considerarse la necesidad de incorporar dichos estudios a las titulaciones sin dicha formación, dada la significación profesional de los elementos comunicativos en el sector turístico.

\section{Referencias bibliográficas}

González-Fernández, A.; Rodríguez-Tapia, S. (2018): "Análisis de los diccionarios como recurso didáctico en el ámbito de la traducción turística desde la lingüística aplicada", M. ${ }^{a}$ Elena Gómez-Parra y Richard Johnstone (coords.), Nuevas perspectivas en educación bilingüe: investigación e innovación, Granada: Editorial Universidad de Granada, 97-104.

Moreno, M. ${ }^{a}$ C.; Rodríguez-Tapia, S. (2015): "La situación de la traducción agroalimentaria en la investigación y la formación en España”, Skopos: Revista Internacional de Traducción e Interpretación, 6: I35- I54.

OECD. (2018). OECD Tourism Trends and Policies 2018. OECD Publishing: Paris. Recuperado de http://dx.doi.org//0.1787/tour-2018-en.

Porlán, R.I (2013), "Entrevista: Ana Ballesteros (COVAP, Area Manager)", Skopos: Revista Internacional de Traducción e Interpretación, 2: 145-154.

TURESPAÑA, Instituto de turismo de España. Boletín trimestral de coyuntura turística. (Coyuntur), 30, primer trimestre de 2018. Ministerio de Energía, Turismo y Agenda Digital. Subdirección General de Conocimiento y Estudios Turísticos. Gobierno de España. NIPO: 086-17-017-6. Recuperado de http://estadisticas.tourspain.es/es-

ES/estadisticas/analisisturistico/coyuntur/trimestrales/Coyuntur\%2030.\%20 Primer\%20trimestre\%202018.pdf. 